rather higher than A-level mathematics, the principal aim to derive the standard distributions which arise in sampling from a Normal distribution. The approach is unusual and intriguing, giving emphasis to the family of Pearson curves (categorised in a new way) and the identification of members of this family by their moment generating functions. The author's claim that this "helps to give a unified setting to all the common distributions " is largely true, especially in respect of limit theorems, although the reader may feel disappointed by the change of approach which the non-existence of certain M.G.F.s entails.

The author uses the phrase, obviously with dislike - "those dreadful overhangs from the days of Victorian statistics": I am unconvinced that he is not following the same path, being fascinated by the discovery and description of relationships between distributions, albeit in mathematical rather than numerical terms. The independent learner, whose modern educational needs are to be met by this series, will find a well-written book full of interesting mathematical manipulations. In itself, however, it is not a book about statistics.

\title{
R. M. CORMACK
}

SinClair, Allan M., Automatic Continuity of Linear Operators (Cambridge University Press, 1976), 92 pp., £2.90.

Theorems on the automatic continuity of functions preserving certain algebraic structures have a natural appeal to mathematicians. Allan Sinclair, who has made notable contributions to this field, is to be commended for putting in permanent form his postgraduate lecture course on automatic continuity given at the University of Edinburgh. Most of the results involve Lebesgue rolling hump arguments; a preliminary chapter attempts to provide some universal technical lemmas and this streamlines the subsequent proofs of a rich variety of theorems, even though the synthesis is not as complete as the author would wish. A brief second chapter treats the problem of intertwining linear operators, i.e. operators $S$ such that $S T=R S$ for some continuous linear operators $T, R$ on Banach spaces, including complete answers when $T, R$ are normal operators on Hilbert spaces. The main chapter deals with homomorphisms of Banach algebras and begins with Johnson's theorem on the uniqueness of complete algebra-norm topology on semi-simple Banach algebras. It also includes two methods of constructing discontinuous derivations, with an example for the disc algebra. The Bade-Curtis results are developed for homomorphisms of $C(\Omega)$ and the chapter culminates in homomorphisms and derivations of $C^{*}$-algebras. The final result reduces the existence of a discontinuous homomorphism of a $C^{*}$-algebra to a Banach algebra to the case when the $C^{*}$-algebra is $C[0,1]$. Just after publication of these notes Dales and Esterle independently have shown that the continuum hypothesis implies the existence of a discontinuous homomorphism of $C[0,1]$. The final chapter expounds the delightful results on the automatic continuity of positive linear functionals on Banach star algebras. There are full references to the literature and research problems are scattered through the notes. Functional analysts will find a lot of good reading in this slim volume, postgraduates will find it a boon.

J. DUNCAN

THOM, RENE, Structural Stability and Morphogenesis: An Outline of a General Theory of Models (Addison-Wesley, 1975), xxv +348 pp., $\$ 22.50$ (cloth), \$13.50 (paper).

Ca tastrophe theory lends itself to applications of a novel type, and a keen interest in these is spread far beyond the circle of experts in this field. Thus the appearance of a book such as the one under review, by so eminent an author, is an event of considerable 
importance. A reviewer (C. W. Kilmister) of the original (1972) French edition wrote "In some sense the only book with which [Thom's book] can be compared is Newton's Principia" and a distinguished biologist (the late $\mathrm{C}$. $\mathrm{H}$. Waddington) expresses in the Foreword to the present translation his conviction "that Thom's book is one of the most original contributions to the methodology of thought in the last several decades". It should perhaps be added that these words of encomium are bestowed on the general spirit of the book rather than on any specific conclusions. ". . the criterion for Thom's speculative applications must come from their stimulating character without trying to prejudge their correctness" (Kilmister), and "... it is likely that not all Thom's suggestions will prove acceptable" (Waddington).

The book is definitely not easy reading. For one thing, few readers will possess the requisite knowledge in both differential topology and the natural sciences, notably biology. For another, the scope of applications is very wide. The word "morphogenesis" in the title refers not only to the process biologists call by this name but more generally to the emergence of new forms due to a discontinuity in the nature of a system. Much of the book is concerned with applications to biology, ranging from the most specific (gastrulation in birds) to the most general (finality in biology, the origin of life), but there are references to hydraulics, physics, geology, and there is a chapter on thought, language, and human societies. In addition to the difficulties inherent in the subject and the scope of the book, there is also the difficulty of following the author's highly intuitive reasoning which at times proceeds in what to the uninitiated appears as leaps and bounds.

Slightly less than one-half of the book is devoted to the development of a species of qualitative dynamics. The brief introductory chapter is philosophical in nature. Ideally, a system is described by a quantitative global model $(M, K, X)$, where $M$ is the space of observables, $K$ a closed subset of $M$ called the catastrophe set, and $X$ a vector field on $M$ defining the evolution of the system. Morphogenesis occurs when the point of $M$ characterising the local state of the system enters $K$. In most situations it is difficult if not at present impossible to construct $(M, K, X)$. The crux of Thom's method is based on the possibility, due to recent advances in differential topology, of constructing a purely geometrical theory of morphogenesis, largely independent of the substratum of forms and the forces generating them.

Next, the concept of a form is analysed. A form is structurally stable if small changes of it result in an equivalent form. The system is now described in terms of a differentiable manifold of forms, and its evolution in terms of differentiable maps. Structurally stable forms are called generic points and constitute an open subset; those forms at which structural stability fails are called bifurcation points and constitute the catastrophe set. Catastrophes can be classified, and there is a remarkably short complete enumeration of catastrophes of the simplest type. This leads to a general morphology, and an investigation of the kinematics and dynamics of forms.

This qualitative dynamics was developed by the author in an attempt to give meaning to the embriologists' concept of morphogenetic field, and to Waddington's chreods. In the author's view, biologists must from the start postulate the existence of local determinism to account for microphenomena, and then attempt to integrate the phenomena into a global structure. The transition from local to global is at the root of the connection with topology. It does not appear to be feasible to indicate adequately, in a brief review, the flavour of the work; at best an enumeration of some of the topics taken up can be attempted. Under local models in embryology are included gastrulation, the morphogenesis of a vertebrate limb, neurulation, and the formation of the spinal chord. Under global models for a living being we find discussions of, e.g. respiration and blood circulation and the nervous system. Then follow models in ultrastructure with cell division, mitosis, cytoplasmic structures. The biological part 
concludes with a chapter on the basic problems of biology including finality, irreversibility of differentiation, the origin of life, and evolution. The final chapter of the book is entitled "From animal to man: thought and language". It contains sections on the animal mind, homo faber, homo loquax, the origin or geometry, art, human play, and the structure of societies.

Even a person lacking in the mental equipment necessary for a full understanding and critical appreciation of this book will find its reading intensely stimulating and thought-provoking. As to the difficulties he encounters reading the book, one remembers that James Hutton's important and novel ideas on geology had to await a secondary presentation by John Playfair before they were fully understood and became effective.

\section{A. ERDÉLYI}

HumPHREYS, J. E., Linear Algebraic Groups (Springer-Verlag, Berlin, 1975), xv +247 pp., $\$ 18.90$.

The author's aim in this book has been to write an introductory text on algebraic groups which would be accessible to workers in associated fields, e.g. finite simple groups, infinite linear groups. This is by no means an easy task but has been accomplished very successfully and I would thoroughly recommend the book to anyone wishing to acquire an understanding of the basic ideas of algebraic groups.

The contents of the book are necessarily very similar to those of Borel's "Linear Algebraic Groups" but do include an additional chapter on Representation and Classification of Semisimple Groups. As does Borel, Humphreys begins by giving an account of the algebraic geometry required. The main differences here are that the terminology of schemes is avoided completely and also only an algebraically closed field $K$ is considered. This latter restriction has some obvious disadvantages but does mean that one is able to obtain an intuitive grasp of the basic concepts much more easily. The treatment in this opening chapter is probably the main single feature contributing to the overall success of the book.

In the remaining chapters the treatment is closer to Borel's, the restriction to an algebraically closed field only becoming important in the later chapters. The book ends with a survey of rationality properties but can, of course, give little indication of methods of proof. However, these results can only be appreciated after obtaining the necessary background in the simpler situation and after reaching this stage those who require these results should be able to tackle the more advanced texts on the subject.

In short, this book fits perfectly into the series of Graduate Texts in Mathematics. It does not give a complete survey of the subject and does not give all the latest results which research workers in the field would want. It is a textbook on an advanced topic which many non-specialists will find extremely useful.

\section{J. TOMKINSON}

GILbERT, R. P. and Weinacht, R. J. (editors), Function Theoretic Methods in Differential Equations (Research Notes in Mathematics, Vol. 8, Pitman Publishing, 1976), iix +309 pp., $£ 9 \cdot 50$.

This book is a collection of papers arising out of an issue of Applicable Analysis which was planned for the 85 th birthday of N. I. Muskhelishvili. The collection is made particularly valuable by the inclusion of several recent works by members of the Georgian Academy of Science of the U.S.S.R. The book is divided into three main sections: (1) Generalizations of Analytic Function Theory, (2) Integral Operators, and (3) Boundary Value Problems. The first section is concerned with various methods for 